\title{
Application of computer technology in medical image processing
}

\author{
Wang Jinguo ${ }^{1, a}$, Wang $\mathrm{Na}^{2, b^{*}}$ (corresponding author),Ma Haichun ${ }^{3, \mathrm{c}}$ \\ ${ }^{1}$ Department of Urology, the First Hospital of Jilin University,China \\ ${ }^{2}$ Department of Anesthesiology, the First Hospital of Jilin University, China \\ ${ }^{3}$ Department of Anesthesiology, the First Hospital of Jilin University, China \\ awangjinguolily@163.com, lilyly12345@163.com, ${ }^{\circ}$ Xiaoben6666@126.com
}

Keywords: computer technology. Medical image. CT. Volume visualization.

Abstract. The computer application is becoming more and more widely. It has been applied in many aspects, such as scientific computing, information processing, artificial intelligence and computer communication. In recent years, the application of computer technology in medicine has become a hot research field, and has received wide attention. This paper mainly introduces the application of computer in medical image processing.

\section{Introduction}

Along with the development of medical imaging technology, and the mutual penetration between computer and communication technology and medical imaging science, medical imaging plays an increasingly important role in modern medical diagnosis[1][2].

Computer is widely used in medicine for the management of computer information management system in hospital[3]. It can collect, transfer, process and feedback the medical information in time[4]. It can also enhance and expand the information function of medical personnel, and enhance the medical personnel to identify and deal with medical information, so as to improve the work efficiency of medical personnel.

\section{Medical image classification and Application}

The medical imaging of medical research and clinical diagnosis are varied. It mainly divided into two categories[5][6]: structural image and functional imaging technology. The former is mainly used to obtain the anatomical structure of the human body image, the clear picture of the human body structure and detailed pathological information can provides the reliable means for clinical medicine and medical science research. CT and MRI is a representative of this kind of structure. The other is based on SPECT and PET's functional imaging technology[7]. Functional imaging can detect the biological activity of human organs, and it will be presented in a functional way. Medical research and clinical diagnosis of many important information are in the form of image, medical is very closely related to the image information[8].

\section{Application of direct control imaging process}

The essence of CT is a kind of image processing technique, which is based on the computer and data processing. Although X-ray and photography can enable people to understand the internal structure of the human body, the location of the lesion can be roughly represented by the tomography. Image enhancement system and electrostatic photography have improved the resolution of X-ray and tomography. But only $\mathrm{CT}$ can solve the problem by using the computer to solve the problem of the interference of the scattered lines and the overlapping images and to make 
a quantitative analysis of the absorption system matrix. With the ordinary X-ray perspective different cross sectional images is in CT technology by measuring the intensity of X-ray detection system instead of as an image receptor film. The X-ray tube and detector system Synchronous rotation motion: The projection value of the data matrix is acquired by the detector, and the image is reconstructed by the inverse projection principle and a certain mathematical model. In short, the role of the computer in the CT system is crucial. It is to complete the measurement data acquisition, image building, image reconstruction, image evaluation and image storage, etc. It is also through the body of the X-ray digital matrix formed by processing, after operation, and become visible image output. Without computer technology, the development of CT equipment is unimaginable.

At present, CT (MR-CT), X-CT (CT), CT and CT were used in the clinic. The difference between MR-CT and X-CT is that MR-CT is based on the human body with nuclear spins of atoms. It can produce magnetic resonance imaging in the applied static magnetic field by the corresponding radio frequency, and also produce the spin core number and relaxation time signal after the radio frequency disappeared. Image is a function of the spin core number and relaxation time. X-CT is based on different tissues of human body with different absorption function, it is the density imaging.

\section{Ultrasonic image application}

Ultrasonic image is one of the four major imaging methods in the current imaging diagnosis. It uses the interaction between ultrasound and biology as imaging basis. It has no ionizing radiation, non radioactive, without contraindication, examination time is short, equipment low price advantage, particularly suitable for inspection and diagnosis of soft tissue and organ motion. The ultrasonic imaging method has one dimension A, M and two dimensions B type. There are also special for measuring blood flow velocity and sound. Its principle is to use the internal emission of the human body after the ultrasonic encounter movement, because the probe and the movement of the body of the interface or blood flow between the relative movement, reflected wave frequency and the frequency of the incident, the Doppler phenomenon and the design of ultrasonic Doppler instrument. The important application of computer in ultrasonic image processing is to establish the position signal and control image. The application of computer in processing position signal and controlling image is also found in CT (U-CT) and nuclear medical image processing ECT and providing a dynamic image, which is used to understand the process of the gamma camera. The function of computer enhanced image contrast can also be applied in clinical medicine, such as cell image, electron microscope, $\mathrm{X}$-ray and infrared image to improve the detection rate of small lesions.

\section{Digital subtraction technique application}

Digital subtraction technique is another successful application of computer in image diagnosis. The digital subtraction is the image before or after using the contrast medium to enter the computer, the image is reconstructed by computer in order to improve the image quality. Commonly used subtraction mode has time, energy and fault method. Digital subtraction technology is developing very fast, now can be done by vascular contrast quantitative subtraction technique. Similar in recent years, the rapid development of magnetic resonance digital subtraction (DSMR). 


\section{Volume visualization technology application}

At present, a new 3D medical image based on volume visualization technology is becoming more and more widely used in the world. The computer visualization technology is developed from the visual technology.

In medical diagnosis, a set of two dimensional CT images of a patient is observed as a routine method for diagnosis of the disease. However, it is very difficult to accurately determine the spatial location, size, geometry, and the spatial relationship between the lesion and the surrounding tissue. Therefore, it is urgent to need a kind of effective tool to complete the 3D reconstruction and 3D display of human organs, soft tissues and lesions. Volume visualization technique is an effective tool to analyze and display the lesions and surrounding tissues. It greatly improves the accuracy and the scientific nature of medical diagnosis.

At present, the United States in accordance with the Department of radiology of the Ahua University of multimedia laboratory provides a virtual hospital. The virtual hospital on the network uses Internet existing communication standards and WWW technology, and the internal and external environment of each department of the whole hospital, in order to reproduce the multimedia knowledge base system as far as possible in the network. It has brought great convenience to doctors, patients, and all of the health care workers. Based on the real hospital department of environmental knowledge base, not only can provide good medical consultation service, but also can provide vivid teaching material for the hospital and other medical personnel in the remote area.

\section{The significance of volume visualization technology in other aspects}

Volume visualization Not only enhances the medical diagnosis level, but also plays an important role in the operation planning and simulation, anatomy education and medical research. Specific performance is to provide the three-dimensional structure of the organ and tissue information, so that the doctor to make a correct judgment. It is to carry out the operation planning and operation process simulation, improve the reliability and safety of the operation. According to the geometrical description of the $3 \mathrm{D}$ reconstruction, the computer aided manufacturing system (CAM) and used to process the human organs. It is also as a tool for medical research and teaching.

The future of medical image not only can "watch", also can realize virtual reality, to create a realistic virtual environment, let the operator in this virtual environment, participate in the $3 \mathrm{D}$ imaging of the human operation and reconstruction activities. The operator is like being in the real world. The technology allows doctors to implement a variety of surgical procedures for patients in the virtual operation room model, the simulation of the operation process, so that doctors can carry out several times before surgery, to help develop the best surgical plan and improve the safety of surgery.

Although this technology has not yet emerged in our country, we believe that with the continuous development of computer technology in the field of medicine, not far in the future, we will see the stereo technology, that is, three-dimensional medical image in the medical industry in China success fully achieved.

\section{References}

[1] Weishar,Peter.Blue Sky: The Art of Computer Animation. 2002.

[2]Miller,G.The Magic Number Seven, Plus or Minus Two: Some Limits on Our Capacity for Processing Information The Psychological Review. . 1956. 
[3]]Mi cha elGS. Review of compression methods for medical images in PACS. International Journal of Medical Informatics . 1998.

[4]Phillip V P.New medical imaging technology. Bio medical Engineering . 1988

[5]VORACEK R,MCADAMS H P,FLOYD C E.Computer Aided Diagnosis of Interstitial Lung

Disease:a Texture Feature Extrac-tion and Classification Approach. Proceedings of SPIE the International Society for Optical Engineering . 1998

[6]TIANG JIE,LIUNingning,ZHUGE Ying.AWindows NTbased3D imaging and analysis system. Proceeding of First Euro\&China Conference on the Information society . 1997.

[7] SHARMAN R,TYLER J M,PIANYKHO L,et al.A fast and ac-curate to method to register medical images using wavelet modulus maxima. Pattern Recognition Letters. 2000.

[8]LUO Shuoqian, LI Xiang.Implementation of mutual information based multi-modality medical image registration. Eng Med Bill Soc Proc22nd Ann Int Conf IEEE . 2000. 